\section{Measurement of Distributed Antenna Systems at 2.4 GHz in a Realistic Subway Tunnel Environment}

\author{
Ke Guan, Student Member, IEEE, Zhangdui Zhong, José I. Alonso, \\ and Cesar Briso-Rodríguez
}

\begin{abstract}
Accurate characterization of the radio channel in tunnels is of great importance for new signaling and train control communications systems. To model this environment, measurements have been taken at 2.4 GHz in a real environment in Madrid subway. The measurements were carried out with four base station transmitters installed in a 2-km tunnel and using a mobile receiver installed on a standard train. First, with an optimum antenna configuration, all the propagation characteristies of a complex subway environment, including near shadowing, path loss, shadow fading, fast fading, level crossing rate (LCR), and average fade duration (AFD), have been measured and computed. Thereafter, comparisons of propagation characteristics in a double-track tunnel (9.8-m width) and a single-track tunnel (4.8-m width) have been made. Finally, all the measurement results have been shown in a complete table for accurate statistical modeling.
\end{abstract}

\section{INTRODUCTION}

Nowadays, railway radio communications are becoming more and more important for train control and signaling. High-quality radio communications permit us to increase the capacity and reliability of subways [1]. Modern subways use communications-based train control systems (CBTCs) for the signaling and control of trains. This system is based on the use of continuous radio communications to communicate with the trains. The next generation of CBTC systems will require high-quality video for trains' control and surveillance. This system is mandatory for automated driving of trains scheduled for year 2020 in Madrid subway.

There are mainly two ways to provide radio coverage inside tunnels, i.e., leaky feeders and distributed antennas [2], [3]. Leaky feeders have been widely used; however, it becomes rather expensive for systems operating at higher carrier frequencies, and maintenance is complex after tunnels have been opened for the traffic [4].

Radio coverage with antennas has a 1/10 deployment cost, compared with leaky feeders, and it has advantages such as higher distance between repeaters, easy maintenance, and no upper frequency limit.

Manuscript received April 27, 2010; revised November 1, 2011; accepted November 25, 2011. Date of publication December 8, 2011; date of current version February 21, 2012. This work was supported in part by the National Science Foundation of China (NSF) under Grant 60830001, by the Program for New Century Excellent Talents in University under Grant NCET-09-0206, by the Beijing NSF 4112048, by the Key Project of the State Key Laboratory of Rail Traffic Control and Safety under Grant RCS2008ZZ006, and by the Fundamental Research Funds for the Central Universities under Grant 2010 JBZ008. The review of this paper was coordinated by Prof. C. P. Oestges.

K. Guan and Z. Zhong are with the State Key Laboratory of Rail Traffic Control and Safety, Beijing Jiaotong University, Beijing 100044, China (e-mail: myecone@hotmail.com).

J. I. Alonso is with the Dpto. SSR. E.T.S.I. Telecomunicación, Universidad Politécnica de Madrid, 28031 Madrid, Spain.

C. Briso-Rodríguez is with the Escuela Universitaria de Ingeniería Técnica de Telecomunicación, Universidad Politécnica de Madrid, 28031 Madrid, Spain.

Color versions of one or more of the figures in this paper are available online at http://ieeexplore.iee. org.

Digital Object Identifier 10.1109/TVT.2011.2178623

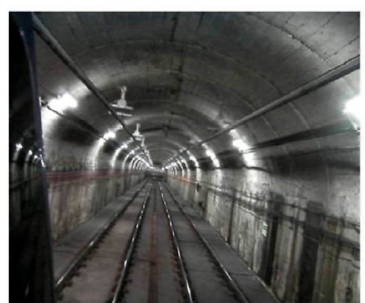

(a)

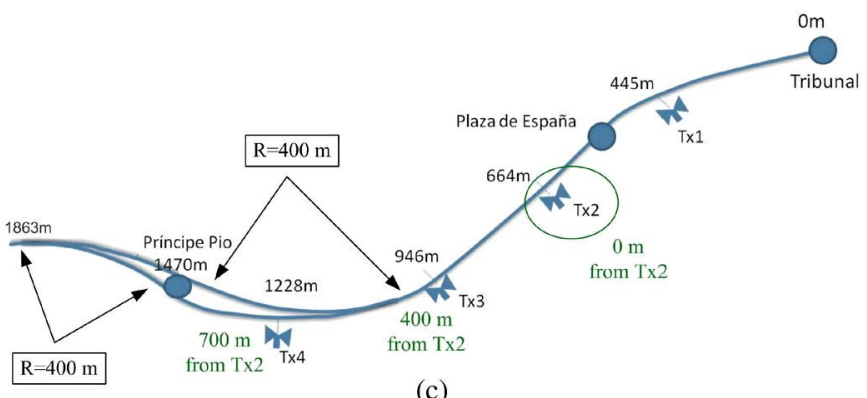

(c)

Fig. 1. (a) Classical arched tunnel used in measurements. (b) Schema of the on-train receiving antenna's position. (c) Skeleton map of the tunnel: TribunalPríncipe Pio.

However, radio planning requires more engineering work, particularly when the communication system must have high-quality service along the entire track [3], [4].

There are considerable differences between tunnels for cars or trains, as well as in the railway environment between subway, light rail trains, or high-speed trains. Moreover, there are important differences between measurements carried out in a realistic and nonrealistic environment, with and without trains. To overcome these problems and meet future needs, a real solution for $2-\mathrm{km}$ tunnels with four base transmitters and three train stations using standard subway trains has been measured. Effects such as near shadowing of long trains, shadow fading, path loss, multimode, and attenuation have been accurately measured and statistically modeled to permit the design with distributed antenna solution, meeting the service quality required for train control systems.

\section{A. Measurement Environment}

The measurements have been carried out in the Line 10 tunnels of Madrid's subway, between Tribunal and Príncipe Pio stations. On this line, the arched shape and construction of the tunnels are the most common in Madrid subway. Fig. 1 shows the testing tunnel, the schema of the on-train receiving antenna's position, and the skeleton map of the tunnels.

As shown in Fig. 1(a), the cross section of testing tunnels is arched, which can be approximately regarded as an equivalent rectangle. Along a 1863-m-long track, four tunnel sections (three wide tunnels and one narrow tunnel) have been selected for measuring with the dimensions $(9.6 \mathrm{~m} \times 6.1 \mathrm{~m}),(9.8 \mathrm{~m} \times 6.2 \mathrm{~m}),(9.7 \mathrm{~m} \times 6.1 \mathrm{~m})$, and $(4.8 \mathrm{~m} \times 5.3 \mathrm{~m})$. Correspondingly, four transmitters are installed at $4 \mathrm{~m}$ above the floor; $25 \mathrm{~cm}$ from the tunnel walls; and 455, 664, 946, and $1228 \mathrm{~m}$ along the track, with different operating frequencies at $2481,2454,2427$, and $2400 \mathrm{MHz}$, respectively. The receiving antennas are in the front and rear cars of the train, $2.2 \mathrm{~m}$ from the nearest wall and $4.5 \mathrm{~m}$ above the track, as shown in Fig. 1(b).

The distance between transmitters is calculated by a computer program using the modal analysis propagation model described in [5]. 


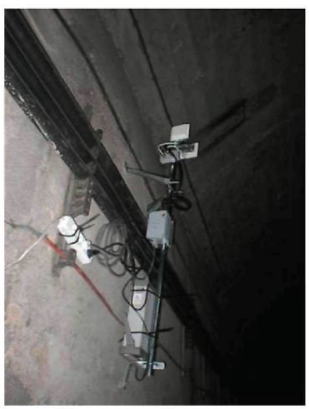

(a)

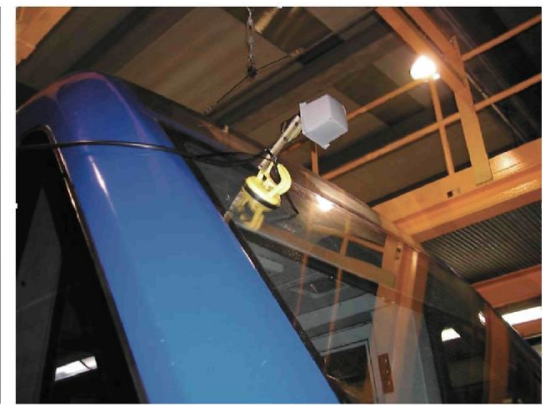

(b)

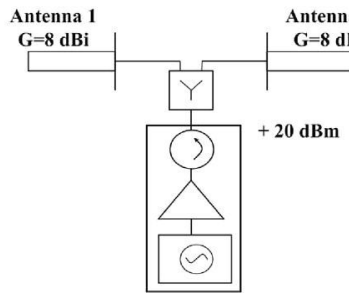

(c)

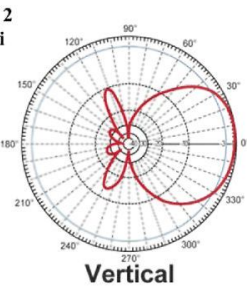

(d)

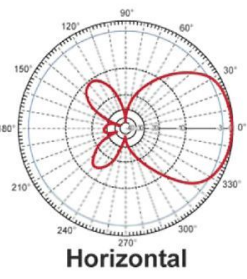

Fig. 2. (a) Test transmitter installed on the tunnel. (b) Antennas' configuration in the cabin of the train. (c) Block diagram of the test transmitter. (d) Antenna patterns.

The network is designed with a moderate overlap of the transmitters. The distance of the overlap depends on the characteristics of the tunnel, the maximum speed of the trains, and the radio modulation system used. The objective is to guarantee that a handover is possible between two neighbor transmitters. We plan to use 2-MHz-bandwidth code-division multiple-access transmitters with a data rate of $250 \mathrm{~kb} / \mathrm{s}$ and the minimum useful signal of $-80 \mathrm{dBm}$. Since the minimum time of the transmitter to do a handover is $4 \mathrm{~s}$, with normal trains for subway with a top speed of $120 \mathrm{~km} / \mathrm{h}(33.3 \mathrm{~m} / \mathrm{s})$, a minimum of $266 \mathrm{~m}(2 \times 133 \mathrm{~m})$ of overlap is required. With a conservative consideration and therefore choosing a safe margin of $10 \mathrm{~dB}$ over the minimum level, the overlap between transmitters is obtained at $500 \mathrm{~m}$.

Detailed parameters of the tunnel in the measurements and location information of transmitters have been supported in Fig. 1(c).

\section{B. Test System}

The test system works from -80 to $-10 \mathrm{dBm}$. (The noise floor is $-90 \mathrm{dBm}$.) The transmitters are proprietary portable equipment that covers the $2.4 / 2.5-\mathrm{GHz}$ band in $1-\mathrm{MHz}$ steps with an output power of $20 \mathrm{dBm}$. It has a 3 -dB power splitter to feed two HyperLink HG2409P antennas (circular polarization with $8-\mathrm{dBi}$ gain), each one pointing to one side of the tunnel.

The receiving system is composed of a spectrum analyzer, a portable computer, a proprietary control software, external antennas, and lownoise amplifiers. The receiving antennas are the same as for the transmitters but vertical polarized with $65^{\circ}$ horizontal and vertical beam width. Near each antenna, there is a $30-\mathrm{dB}$ preamplifier to improve noise figure and reduce coaxial feeder loss. The two-antenna configuration requires that each antenna is first preamplified and then combined with a $3-\mathrm{dB}$ power splitter. The objective is to get the same input level at the input of the spectrum analyzer.

Fig. 2 shows a transmitter installed on the wall of the tunnel, one of the directive antennas installed in the front of the train, the block diagram of one transmitter, and the radiation pattern of the antennas used for both the transmitter and the receiver.

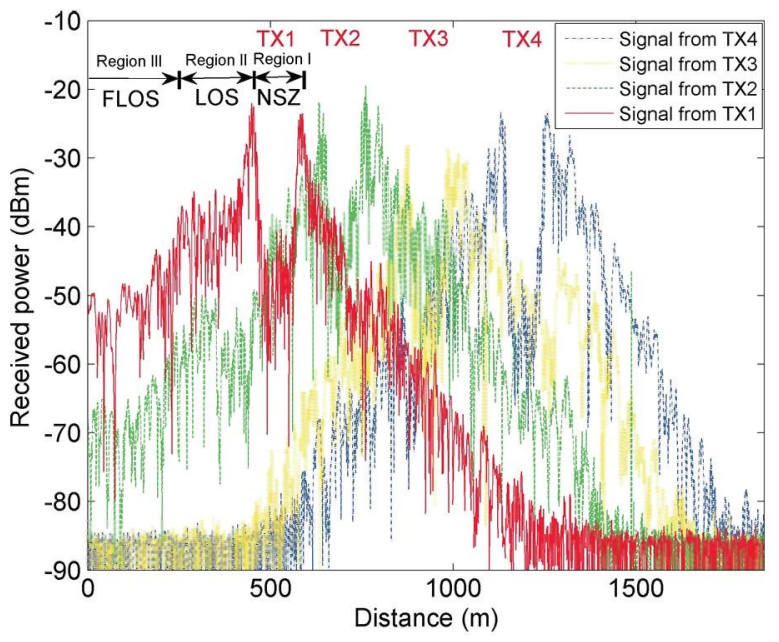

Fig. 3. Received power in the tunnel with four transmitters and a description of different regions.

The receiver is deployed in the locomotive cabin of one of the trains. The trains used are standard Madrid subway trains with four cars and 60-m length.

The test system is able to take 100 measurement/s. With this recording speed at $2.4 \mathrm{GHz}$ and for a maximum train speed of $20 \mathrm{~km} / \mathrm{h}$, we have obtained a rate of one measurement per 0.44 wavelength.

\section{Measurements And Propagation}

The objective of the measurements is to provide data to a real complex subway environment under real conditions. With this objective, the following two different scenarios have been tested:

1) referenced configuration with one antenna in the front and one in the rear;

2) narrow tunnel and wide tunnel.

\section{A. Propagation Measurements With Referenced Antenna Configuration}

The first group of measurements have been taken using a configuration of two antennas, with one installed in the front and one in the rear car of the train. It is known that this configuration provides good results; therefore, it is widely used in railway systems. With this configuration, the signal received by each antenna is strongly uncorrelated due to the directivity, long distance between antennas, and strong self-shadowing of the train.

The received signal power from four transmitters is shown in Fig. 3, which shows very good propagation in the entire tunnel. CBTC communications systems require a minimum signal level of $-70 \mathrm{dBm}$ to operate. Therefore, we can see that the coverage is continuous with strong overlapping between neighbor cells, so that the distance between transmitters can be increased.

With the measurement results, statistics of propagation can be done.

1) Near-Shadowing Phenomenon: An important phenomenon has been observed: when the train is passing in front of the transmitter, the received signal power suffers deep fading, and propagation has strong multipath. We call this phenomenon near shadowing; a corresponding near-shadowing zone (NSZ) is shown in Fig. 3, and the effect is modeled in [6].

When the train is passing in front of the transmitter, the signal is blocked by the train because the subway tunnels are very narrow and the trains are very long $(60-20 \mathrm{~m})$. At $2.4 \mathrm{GHz}$, antennas are directive, and in narrow tunnels, it is impossible to get line of sight (LOS) with 


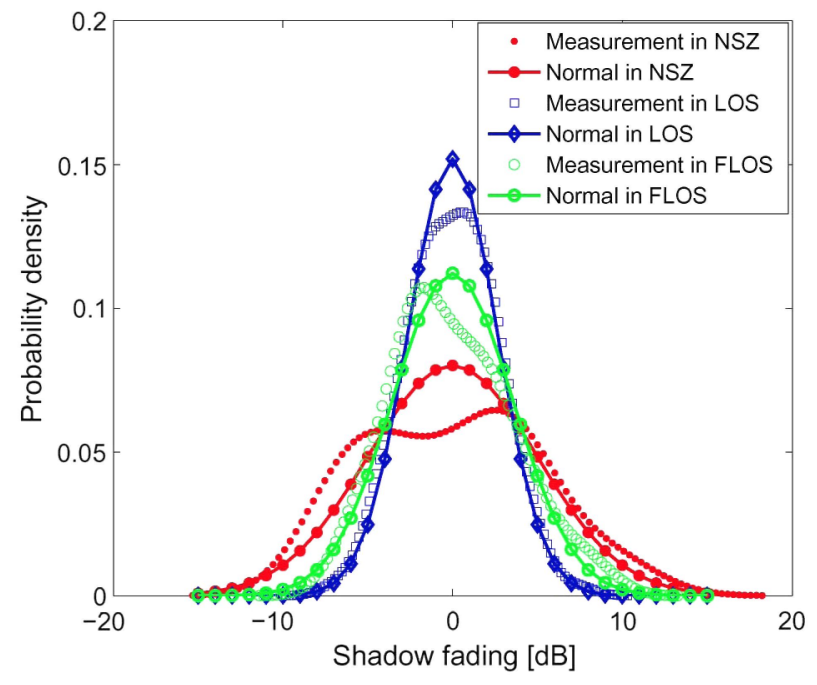

Fig. 4. Statistical modeling of fading in the three regions.

the transmitter, even if the antennas are located in the upper part of the train.

2) Path Loss: A statistical model [7] has been chosen to analyze the path-loss exponent, i.e.,

$$
P L(d)[d B]=P L\left(d_{0}\right)+10 n \log \left(d / d_{0}\right)+X_{\sigma}
$$

where $n$ is the path-loss exponent; $d_{0}$ is the reference distance, and $1 \mathrm{~m}$ is used inside the tunnel [8]. $P L(d)$ is the ensemble average of all possible path-loss values for a given distance $d$.

3) Shadow Fading: The most common model, which has been empirically confirmed for shadow fading is lognormal distribution $X_{\sigma}$ [9]. $X_{\sigma}$ is a zero-mean Gaussian distributed random variable (in decibels) with standard deviation $\sigma$ (in decibels).

In Fig. 3, the measurement result has shown good coverage and has allowed us to analyze the shadow-fading probability density function by defining three different regions [10].

1) Region I (NSZ): When the train is passing by the transmitter, the LOS between the transmitter and receiving antennas is blocked by the train. As there is no LOS component, the multipath propagation is dominant in this region. This renders $\sigma$ in this region bigger than in the other two regions.

2) Region II (LOS): When the train has passed but is still near the transmitter, there is a clear LOS propagation resulting in the smallest $\sigma$.

3) Region III [Far LOS (FLOS)]: There is no LOS because the tunnel has a smooth curve, as shown in Fig. 1(c). Hence, the direct wave is more attenuated, and the propagation fading is higher farther from the transmitter. The reflected wave is more significant than the direct wave [10]. Thus, $\sigma$ is smaller than that in Region I but larger than that in Region II.

As shown in Fig. 4, we statistically modeled fading in the three regions using normal distribution. It can be seen that the fitting is very good in LOS and FLOS regions and a little bit worse for NSZ. These fittings have been done using Easy Fit [11] with a good result. We have decided to fit the three zones with Gaussian functions to simplify comparison among them.

In a normal network, the train transceivers make handovers between different base-station transmitters along the entire track; therefore, one overall measurement of the network quality is the power sum enveloped from the signal of all the transmitters deployed on the track.
TABLE I

Global Measurement Results

\begin{tabular}{|c|c|c|c|c|}
\hline \multicolumn{5}{|c|}{$\begin{array}{l}\text { Propagation in tunnels } \\
\text { with referenced antennas configuration }\end{array}$} \\
\hline & \multirow[t]{2}{*}{$\begin{array}{l}\text { Power } \\
\text { received }\end{array}$} & \multicolumn{3}{|c|}{$\begin{array}{l}\text { Distance between successive } \\
\text { fadings with depth of }\end{array}$} \\
\hline & & $0 \mathrm{~dB}$ & $5 \mathrm{~dB}$ & $10 \mathrm{~dB}$ \\
\hline Mean & $-53.2 \mathrm{dBm}$ & $3.5 \mathrm{~m}$ & $21.9 \mathrm{~m}$ & $164.4 \mathrm{~m}$ \\
\hline Median & $-49.3 \mathrm{dBm}$ & $3 \mathrm{~m}$ & $11 \mathrm{~m}$ & $73 \mathrm{~m}$ \\
\hline Std & $5.8 \mathrm{~dB}$ & $1.7 \mathrm{~m}$ & $25.5 \mathrm{~m}$ & $164.9 \mathrm{n}$ \\
\hline Distribution & \multicolumn{4}{|c|}{ Gaussian (in $\mathrm{dB}$ ) } \\
\hline Distance & \multicolumn{4}{|c|}{$0-1863 \mathrm{~m}$} \\
\hline $\begin{array}{l}\text { Fading } \\
\text { depth } \\
\text { (dB) }\end{array}$ & \multicolumn{2}{|c|}{$\begin{array}{c}\text { AFD } \\
\text { (wavelengths) }\end{array}$} & \multicolumn{2}{|c|}{$\begin{array}{c}\text { LCR } \\
\text { (wavelengths }{ }^{-1} \text { ) }\end{array}$} \\
\hline 5 & \multicolumn{2}{|c|}{0.008} & \multicolumn{2}{|c|}{0.098} \\
\hline 10 & \multicolumn{2}{|c|}{0.001} & \multicolumn{2}{|c|}{0.007} \\
\hline \multicolumn{5}{|c|}{ Fast fading of the received signal power: Nakagami- $m$} \\
\hline Median & Mean & Std & Min & Max \\
\hline 4.3 & 6.5 & 6.7 & 1.1 & 39.9 \\
\hline \multirow[t]{2}{*}{ Zone } & $\begin{array}{l}\text { Reg. I } \\
\text { Near }\end{array}$ & & . II & Reg. II \\
\hline & shadowing & & OS & FLOS \\
\hline \multicolumn{5}{|c|}{ Shadow fading of the received signal power (TX1) } \\
\hline Distribution & $\begin{array}{l}\text { Normal } \\
\text { (in dB) }\end{array}$ & & & $\begin{array}{l}\text { Normal } \\
\text { (in } \mathrm{dB} \text { ) }\end{array}$ \\
\hline Std & $5.0 \mathrm{~dB}$ & & $\mathrm{~dB}$ & $3.6 \mathrm{~dB}$ \\
\hline \multicolumn{5}{|c|}{$\begin{array}{l}\text { Fast fading of the received signal power (TX1) } \\
\text { Nakagami }\end{array}$} \\
\hline$m$ & 0.8 & & .8 & 2.4 \\
\hline \multicolumn{3}{|c|}{ Tunnel dimension } & Wide & Narrow \\
\hline \multirow{2}{*}{\multicolumn{3}{|c|}{$\begin{array}{l}\text { Mean of near shadowing depth } \\
\text { Length of near shadowing zone }\end{array}$}} & $21.4 \mathrm{~dB}$ & $32.1 \mathrm{~d}$ \\
\hline & & & $\begin{array}{r}\text { two } \\
\text { the tra }\end{array}$ & imes \\
\hline \multicolumn{3}{|c|}{ Std of shadow fading $\sigma$} & $2.75 \mathrm{~dB}$ & $4.17 \mathrm{~dB}$ \\
\hline \multicolumn{3}{|c|}{$\begin{array}{l}\text { Path loss exponent } n \\
\text { (real curved tunnel) }\end{array}$} & 5.49 & 7.13 \\
\hline \multicolumn{3}{|c|}{$\begin{array}{l}\text { Attenuation rate } \\
\text { (real curved tunnel) }\end{array}$} & $\begin{array}{c}69.5 \\
\mathrm{~dB} / \mathrm{km}\end{array}$ & $\begin{array}{c}85.9 \\
\mathrm{~dB} / \mathrm{km}\end{array}$ \\
\hline \multicolumn{3}{|c|}{ Nakagami- $m$} & 5.4 & 4.4 \\
\hline
\end{tabular}

The signal deviation of the power sum is the sum of four lognormal distributions of the four transmitters, and according to the central limit theorem, this number is high to reach a Gaussian distribution (in decibels) with a standard deviation of $5.8 \mathrm{~dB}$, providing a good root-mean-square quality of signal.

4) Fast Fading: Nakagami- $m$ distribution has been found to be a very good fitting for mobile radio channel fast fading. Parameter $m$, which indicates the severity of the amplitude fading, is estimated by using the moment-based method in all the testing and comparison cases. According to Lee's Criteria, we choose the window with a length of 91 samples to separate the local mean from the fast fading data. Details are shown in Table I.

5) LCR and AFD: Level crossing rate (LCR) and average fade duration (AFD) with respect to various fading depths have been deduced from measured values. Details are given in Table I.

\section{B. Narrow Tunnel and Wide Tunnel}

Subway tunnels frequently split into two single-track tunnels when arriving to large stations; therefore, it is important to model propagation in these situations. We have tested inside an arch-shaped wide tunnel $(9.8 \mathrm{~m} \times 6.2 \mathrm{~m})$ and a narrow tunnel $(4.8 \mathrm{~m} \times 5.3 \mathrm{~m})$. 


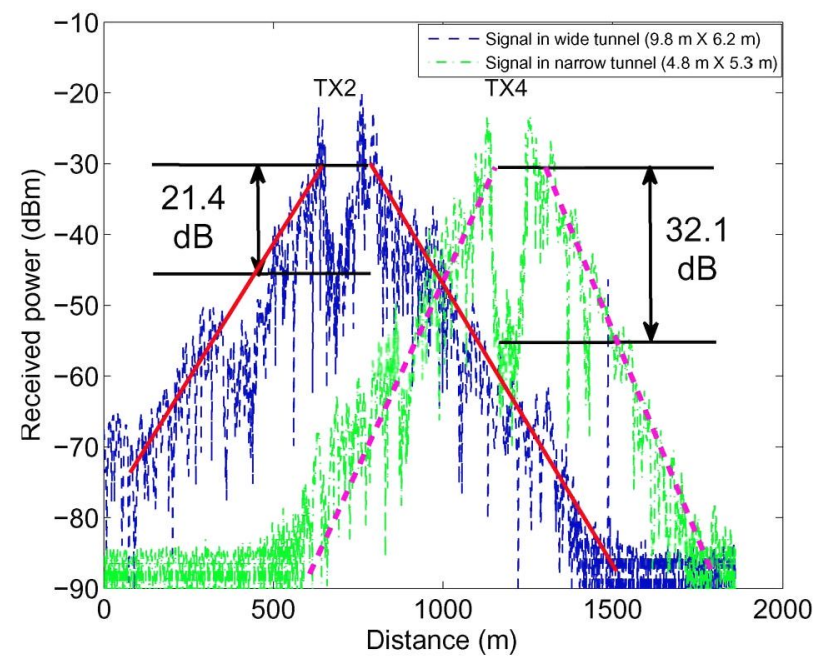

Fig. 5. Received signal power between the wide tunnel and the narrow tunnel.

Fig. 5 shows the power received from two transmitters, i.e., one (TX2) in a double-track wide tunnel and the other one (TX4) in a single-track narrow tunnel. The wide tunnel, as shown in Fig. 1(c), goes from 0 to $1000 \mathrm{~m}$, and the narrow tunnel is $500 \mathrm{~m}$ in length, starting from point $1000 \mathrm{~m}$ and ending on point $1500 \mathrm{~m}$. At position $1470 \mathrm{~m}$ is a subway station.

1) Near-shadowing effect: The received signal suffers a severe attenuation of up to $21.4 \mathrm{~dB}$ inside the wide tunnel $(9.8 \mathrm{~m} \times$ $6.2 \mathrm{~m})$ and $32.1 \mathrm{~dB}$ inside the narrow tunnel $(4.8 \mathrm{~m} \times 5.3 \mathrm{~m})$. These values are high and must be carefully considered in the design of a network.

2) Path loss: Fig. 5 demonstrates the different situations of path loss in tunnels with various widths. Corresponding path loss exponents have been extracted and shown in Table I.

3) Attenuation rate: As shown in Fig. 5, the attenuation slopes are $69.5 \mathrm{~dB} / \mathrm{km}$ for the wide tunnel and $85.9 \mathrm{~dB} / \mathrm{km}$ for the narrow tunnel.

4) Shadow fading deviation: In the measurement, shadow fading deviation $\sigma$ is $2.75 \mathrm{~dB}$ for the wide tunnel and $4.17 \mathrm{~dB}$ for the narrow tunnel.

5) Fast fading: The Nakagami- $m$ distribution's parameters $m$ is shown in Table I.

\section{CONCLUSION}

A complete group of measurements made at $2.4 \mathrm{GHz}$ has been reported. Measurements have been focused on propagation under real conditions with a long test track, typical tunnels, stations in the middle, real transmitters, and commercial trains. The test network deployed is very similar to a real network and has been used to validate antenna solutions for CBTC.

One interesting new result is the near-shadowing effect created by the train carriages when passing the base station. This effect has been clearly observed with attenuation up to $31.2 \mathrm{~dB}$ and lasting two times the length of the train or up to several seconds, depending on train speed.

A group of important comparisons of propagation between wide tunnel $(9.8 \mathrm{~m} \times 6.2 \mathrm{~m})$ and narrow tunnel $(4.8 \mathrm{~m} \times 5.3 \mathrm{~m})$ with the same conditions has been made. Near shadowing, shadow fading, path loss, fast fading, and attenuation rate have been measured and analyzed.
The results are a complete set of coefficients and comparisons suitable for system simulation and network design of antenna-based communications in tunnel environments. A standard subway line has been measured and analyzed under real conditions, with curves, trains, and all the elements.

\section{ACKNOWLEDGMENT}

The authors would like to thank the Madrid Subway Carrier (METRO) and Dimetronic Invensys Spain for their help, management, and support in conducting these complex measurements.

\section{REFERENCES}

[1] IEEE Standard Method for CBTC Performance and Functional Requirements, IEEE Std. 1474, 2004.

[2] P. Delogne, "Basic mechanisms of tunnel propagation," Radio Sci. vol. 11, no. 4, pp. 295-303, Apr. 1976.

[3] R. J. Jakubowski, "Results of distributed antenna and leaky feeder systems tests at $800 \mathrm{MHz}$ in Washington D.C. metro system tunnels," in Proc. IEEE 44th Veh. Technol. Conf., 1994, vol. 2, pp. 1113-1116.

[4] "Railway radio propagation including aspects of transmission of safety information," ERRI Committee, Utrecht, The Netherlands, Tech. Rep. ERRI A 175/RP2, Jul. 1992.

[5] C. Briso-Rodriguez, J. M. Cruz, and J. I. Alonso, "Measurements and modeling of distributed antenna systems in railway tunnels," IEEE Trans Veh. Technol., vol. 56, no. 5, pp. 2870-2879, Sep. 2007.

[6] K. Guan, Z. D. Zhong, B. Ai, and C. Briso-Rodriguez, "Measurement and modeling of subway near shadowing phenomenon," in Proc. CHINACOM, Beijing, China, 2010, pp. 1-5.

[7] S. T. Rappaport, "Mobile radio propagation: Large-scale path loss," in Wireless Communications C Principles and Practice. Upper Saddle River, NJ: Pearson Educ., 2002, ch. 3, pp. 122-123.

[8] Y. P. Zhang and Y. Hwang, "Measurements and statistical modelling of $900 \mathrm{MHz}$ radio propagation channels for microcellular and personal communications in tunnels," Wireless Pers. Commun., vol. 7, no. 1, pp. 25-39, May 1998.

[9] G. L. Stuber, Principles of Mobile Communication. Boston, MA: Kluwer, 1996.

[10] H. Kwon, Y. Kim, and B. Lee, "Characteristics of radio propagation channels in tunnel environments: A statistical analysis," in Proc. IEEE Antennas Propag. Symp., 2004, vol. 3, pp. 2995-2998.

[11] K. Schittkowski, "EASY-FIT: A software system for data fitting in dynamical Systems," Struct. Multidisciplinary Optim., vol. 23, no. 2, pp. 153 169, Mar. 2002. 